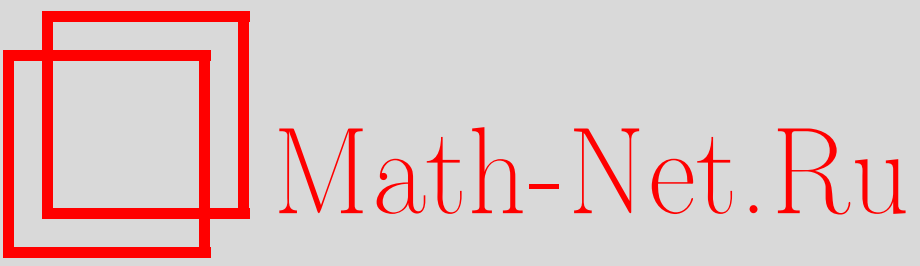

В. Ке, М. А. Чеботарь, О биаддитивных отображениях некоторых алгебр Ли, УМH, 2003, том 58, выпуск 1, 187-188

DOI: https://doi.org/10.4213/rm601

Использование Общероссийского математического портала Math-Net.Ru подразумевает, что вы прочитали и согласны с пользовательским соглашением

http://www.mathnet.ru/rus/agreement

Параметры загрузки:

IP: 52.23 .180 .231

26 апреля 2023 г., 14:37:47 


\title{
О БИАДДИТИВНЫХ ОТОБРАЖЕНИЯХ НЕКОТОРЫХ АЛГЕБР ЛИ
}

\author{
B.- $\Phi$. Ke, M. A. ЧЕБОтАРЬ
}

Биаддитивные отображения первичных колец $f$, удовлетворяющие тождеству $[f(x, x), x]=0$, были описаны Брешаром в работе [1]. Этот резултат имеет многочисленные приложения (см. [2]). В частности, он сыграл ключевую роль в решении известной проблемы Херстейна [1]. Нетрудно заметить, что Брешар описал симметрические биаддитивные отображения первичных колец $f$, удовлетворяющие тождеству $[f(u, v), w]=f([u, w], v)+f(u,[v, w])$. В этой работе мы рассмотримпроизвольные биаддитивные отображения некоторых алгебр Ли, удоволетворяющие этому условию.

Мы будем использовать понятия максимального правого кольца частных и расширенного центроида Мартиндейла, с которыми можно ознакомиться в книге [3].

Теорема 1. Пусть $R$ - первичное кольио, $Q$ - максимальное правое кольцо частных кольиа $R$ и $C$ - расширенный чентроид Мартиндейла. Пусть $L$ - алгебра Ли, и пусть $f: L^{2} \rightarrow Q-$ биаддитивное отображение, удовлетворяющее тождеству

$$
[f(u, v), w]=f([u, w], v)+f(u,[v, w]) \quad \text { для } \quad \text { всех } \quad u, v, w \in L .
$$

Предположим, что выполнено одно из условий:

(1) $L=R$ и $L$ содержит алгебрачческий әлемент степени $\geqslant 4$ над $C$;

(2) $L$ - нечентральный лиевский идеал кольца $R$ и $L$ содержит алгебрачческий әлемент степени $\geqslant 5$ над $C$;

(3) $R$ - кольцо с инволюцией, $L$ - алгебра Ли кососимметрических әлементов и $L$ содержит алгебраический элемент степени $\geqslant 10$ над $C$;

(4) $R$ - кольцо с инволючией, $L$ - лиевский идеал алгебры Ли кососимметрических әлементов и L содержит алгебраический әлемент степени $\geqslant 11$ над $C$.

Тогда существуют $\lambda_{1}, \lambda_{2} \in C$, аддитивнье отображения $\mu_{1}: L \rightarrow C, \mu_{2}: L \rightarrow C u$ биаддитивное отображение $\nu: L^{2} \rightarrow C$ такие, что

$$
f(u, v)=\lambda_{1} u v+\lambda_{2} v u+\mu_{1}(u) v+\mu_{2}(v) u+\nu(u, v)
$$

для всех $u, v \in L$. Более того,

$$
\mu_{1}([L, L])=\mu_{2}([L, L])=0 \quad u \quad \nu([u, w], v)+\nu(u,[v, w])=0
$$

для всех $u, v, w \in L$.

Доказательство следующей теоремы опирается на результаты, использующие понятие $d$-свободных подмножеств (см. [4], [5]).

Теорема 2. Пусть $F$ - коммутативное кольцо с $1, Q-F$-алгебра с 1 и иентром $C$. Пусть $L-4$-свободная лиевская подалгебра алгебрь $Q$, и пусть $f: L^{2} \rightarrow Q-$ биаддитивное отображение, удовлетворяющее тождеству

$$
[f(u, v), w]=f([u, w], v)+f(u,[v, w]) \quad \text { для всех } \quad u, v, w \in L .
$$

Тогда существуют $\lambda_{1}, \lambda_{2} \in C$, аддитивнье отображения $\mu_{1}: L \rightarrow C, \mu_{2}: L \rightarrow C u$ биаддитивное отображение $\nu: L^{2} \rightarrow C$ такие, что

$$
f(u, v)=\lambda_{1} u v+\lambda_{2} v u+\mu_{1}(u) v+\mu_{2}(v) u+\nu(u, v)
$$

для всех $u, v \in L$. Более того,

$$
\mu_{1}([L, L])=\mu_{2}([L, L])=0 \quad u \quad \nu([u, w], v)+\nu(u,[v, w])=0
$$

для всех $u, v, w \in L$. 
ДокАЗАТЕльСтво. Пусть $p: L^{3} \rightarrow Q$ задано по правилу

$$
p(u, v, w)=[f(u, v), w]+[f(v, w), u]+[f(w, u), v] \text { для всех } u, v, w \in L .
$$

Используя тождество Якоби, мы имеем

$$
\left[p\left(x_{1}, x_{2}, x_{3}\right), x_{4}\right]-\left[p\left(x_{2}, x_{3}, x_{4}\right), x_{1}\right]+\left[p\left(x_{3}, x_{4}, x_{1}\right), x_{2}\right]-\left[p\left(x_{4}, x_{1}, x_{2}\right), x_{3}\right]=0
$$

для всех $x_{i} \in L, 1 \leqslant i \leqslant 4$. Ввиду теоремы 1.2 из [5], $p$ - это полиаддитивный квазиполином степени $\leqslant 3$. Применяя снова теорему 1.2 из [5] к тождеству $(2)$, мы заключаем, что $f-$ это полиаддитивный квазиполином степени $\leqslant 2$, т.е.

$$
f(u, v)=\lambda_{1} u v+\lambda_{2} v u+\mu_{1}(u) v+\mu_{2}(v) u+\nu(u, v) \text { при всех } u, v \in L
$$

для некоторых $\lambda_{1}, \lambda_{2} \in C$, аддитивных отображений $\mu_{1}: L \rightarrow C, \mu_{2}: L \rightarrow C$ и биаддитивного отображения $\nu: L^{2} \rightarrow C$. Согласно (1)

$$
\begin{aligned}
{\left[\lambda_{1} u v\right.} & \left.+\lambda_{2} v u+\mu_{1}(u) v+\mu_{2}(v) u+\nu(u, v), w\right] \\
= & \lambda_{1}[u, w] v+\lambda_{2} v[u, w]+\mu_{1}([u, w]) v+\mu_{2}(v)[u, w]+\nu([u, w], v) \\
& +\lambda_{1} u[v, w]+\lambda_{2}[v, w] u+\mu_{1}(u)[v, w]+\mu_{2}([v, w]) u+\nu(u,[v, w])
\end{aligned}
$$

Используя теорему 1.1 из [5], мы получаем

$$
\mu_{1}([u, w])=\mu_{2}([v, w])=0 \text { и } \mu_{2}([u, w], v)+\mu_{2}(u,[v, w])=0
$$

для всех $u, v, w \in L$. Доказательство завершено.

Теорема 1 является следствием теоремы 2, теорем 2.4 и 2.20 из [4] и теоремы 1.1 из [6].

\section{СПИСОК ЛИТЕРАТУРЫ}

[1] M. Brešar // Trans. Amer. Math. Soc. 1993. V. 335. № 2. P. 525-546. [2] M. Brešar // Contemp. Math. 2000. V. 259. P. 93-109. [3] K. I. Beidar, W.S. Martindale, A. V. Mikhalev. Rings with Generalized Identities. New York: Dekker, 1996. [4] K.I. Beidar, M. A. Chebotar // Comm. Algebra. 2000. V. 28. №8. P. 3925-3951. [5] K. I. Beidar, M. A. Chebotar // Comm. Algebra. 2000. V. 28. №8. P. 3953-3972. [6] K. I. Beidar, M. Brešar, M. A. Chebotar, W.S. Martindale // Comm. Algebra. 2000. V. 28. № 7. P. 3169-3183. 